\title{
Development of a patient-derived xenograft model of glioblastoma via intravitreal injection in mice
}

\author{
Jooyoung Lee', Dong Hyun Jo $\mathbb{1}^{2,3}$, Jin Hyoung Kim ${ }^{2,3}$, Chang Sik Cho ${ }^{2,3}$, Jiwon Esther Han', Yona Kim', \\ Hyoungwoo Park', Seung Ho Yoo ${ }^{2}$, Young Suk Yü, ${ }^{2,4}$ Hyo Eun Moon ${ }^{1}$, Hye Ran Park', Dong Gyu Kim 1,5, \\ Jeong Hun Kim ${ }^{2,3,4,6}$ and Sun Ha Paek (1) 1,5,7,8
}

\begin{abstract}
Currently, the two primary patient-derived xenograft (PDX) models of glioblastoma are established through intracranial or subcutaneous injection. In this study, a novel PDX model of glioblastoma was developed via intravitreal injection to facilitate tumor formation in a brain-mimicking microenvironment with improved visibility and fast development. Glioblastoma cells were prepared from the primary and recurrent tumor tissues of a 39-year-old female patient. To demonstrate the feasibility of intracranial tumor formation, U-87 MG and patient-derived glioblastoma cells were injected into the brain parenchyma of Balb/c nude mice. Unlike the U-87 MG cells, the patient-derived glioblastoma cells failed to form intracranial tumors until 6 weeks after tumor cell injection. In contrast, the patientderived cells effectively formed intraocular tumors, progressing from plaques at 2 weeks to masses at 4 weeks after intravitreal injection. The in vivo tumors exhibited the same immunopositivity for human mitochondria, GFAP, vimentin, and nestin as the original tumors in the patient. Furthermore, cells isolated from the in vivo tumors also demonstrated morphology similar to that of their parental cells and immunopositivity for the same markers. Overall, a novel PDX model of glioblastoma was established via the intravitreal injection of tumor cells. This model will be an essential tool to investigate and develop novel therapeutic alternatives for the treatment of glioblastoma.
\end{abstract}

\section{Introduction}

Patient-derived xenograft (PDX) models of glioblastoma are based on the subcutaneous or intracranial injection of tumor cells into immunocompromised mice $^{1}$. These models have been valuable tools to investigate tumor characteristics $^{2,3}$ and the potential therapeutic efficacies of various treatment options ${ }^{1,4-8}$. However, via subcutaneous injection, PDX tumors develop rapidly but are

\footnotetext{
Correspondence: Jeong Hun Kim (steph25@snu.ac.kr) or Sun Ha Paek (paeksh@snu.ac.kr)

${ }^{1}$ Department of Neurosurgery, Seoul National University Hospital, Seoul 03080, Republic of Korea

${ }^{2}$ Fight against Angiogenesis-Related Blindness (FARB) Laboratory, Clinical Research Institute, Seoul National University Hospital, Seoul 03080, Republic of Korea

Full list of author information is available at the end of the article.

These authors contributed equally: Jooyoung Lee, Dong Hyun Jo
}

confined within the subcutaneous space, which is quite different from the brain microenvironment. In contrast, through intracranial injection, tumors experience the brain microenvironment in which glioblastoma is enclosed, but tumor formation and the evaluation of therapeutic efficacy often require time. Considering that the median survival of glioblastoma patients is less than 15 months $^{9-11}$, it is necessary to establish a PDX model of glioblastoma that can be performed more rapidly and mimic the brain microenvironment as much as possible.

In this context, it is remarkable that the retina in the eye and the brain share several neurovascular characteristics in common. First, the retina is composed of neuronal cell layers in which multiple synapses are formed among various neuronal cell types ${ }^{12}$. Second, microvascular endothelial cells in both the brain and the retina form

\section{(c) The Author(s) 2019}

(c) (i) Open Access This article is licensed under a Creative Commons Attribution 4.0 International License, which permits use, sharing, adaptation, distribution and reproduction c. in any medium or format, as long as you give appropriate credit to the original author(s) and the source, provide a link to the Creative Commons license, and indicate if changes were made. The images or other third party material in this article are included in the article's Creative Commons license, unless indicated otherwise in a credit line to the material. If material is not included in the article's Creative Commons license and your intended use is not permitted by statutory regulation or exceeds the permitted use, you will need to obtain permission directly from the copyright holder. To view a copy of this license, visit http://creativecommons.org/licenses/by/4.0/. 
blood-neural barriers with surrounding cells, including pericytes and astrocytes ${ }^{13,14}$. Third, other cellular components of the tumor microenvironment of glioblastoma, including microglia and immune cells, are quite similar between the brain and retina ${ }^{15-17}$.

In this study, a novel PDX model of glioblastoma was established through the intravitreal injection of tumor cells. Unlike U-87 MG cells, these cells from primary and recurrent glioblastoma did not form intracranial tumors. In contrast, intravitreally administered patient-derived cells formed intraocular tumors facing the retinal neuronal tissue within 4 weeks. These in vivo tumors exhibited positivity for glial fibrillary acidic protein (GFAP), vimentin, and nestin, matching the positivity of the original tumors. Furthermore, the cells isolated from in vivo tumors retained the morphological and molecular characteristics of their parental cells.

\section{Materials and methods Primary culture}

Primary samples were obtained from a 39-year-old female patient and an additional four patients (demographic features are summarized in Suppl. Table 1) with glioblastoma after approval from the Institutional Review Board at Seoul National University Hospital (IRB No. H1009-025-331). After collection during tumor resection, the samples were placed into Hank's Balanced Salt Solution (HBSS) containing calcium and magnesium and then chopped with a surgical blade. The tissue samples were subsequently centrifuged at $1100 \mathrm{rpm}$ for $4 \mathrm{~min}$, rinsed with PIPES buffer, and resuspended in phosphatebuffered saline (PBS) containing trypsin-EDTA at $37^{\circ} \mathrm{C}$. Then, the tissue samples were digested with DNase I (20 $\mathrm{U} / \mathrm{mL}$ ) on a rocking shaker for $90 \mathrm{~min}$ at $37^{\circ} \mathrm{C}$, resuspended in Dulbecco's modified Eagle's media (DMEM) containing $10 \%$ fetal bovine serum (FBS), and centrifuged at $1100 \mathrm{rpm}$ for $4 \mathrm{~min}$. After centrifugation, the resuspended cells were filtered through a $40-\mu \mathrm{m}$ cell strainer and seeded in a culture flask. The cells from the primary and recurrent tumors of the 39-year-old female patient were designated GBL-28 and GBL-37, respectively. Glioblastoma cells from tumors that formed after intravitreal injection in mice were isolated using the same protocol.

\section{Animals}

Six-week-old male Balb/c nude mice were purchased from Central Laboratory Animals and maintained under a 12-hour dark/light cycle. All animal experiments were performed in accordance with the Association for Research in Vision and Ophthalmology statement for the use of animals in ophthalmic and vision research and approved by the Institutional Animal Care and Use Committees of both Seoul National University and Seoul National University Hospital.

\section{Cells}

U-87 MG cells (cat. no. HTB-14, ATCC) and patientderived glioblastoma cells (GBL-15, GBL-26, GBL-28, GBL-30, GBL-37, and GBL-211) were maintained in DMEM with $10 \% \mathrm{FBS}$ at $37^{\circ} \mathrm{C}$ in a humidified atmosphere of $95 \%$ air and $5 \% \mathrm{CO}_{2}$.

\section{Orthotopic transplantation of glioblastoma cells}

After deep anesthesia, mice were positioned in a stereotactic frame (David Kopf Instruments). A small craniectomy was performed at $2-3 \mathrm{~mm}$ from the midline and $1 \mathrm{~mm}$ anterior to the coronal suture. Glioblastoma cells (U-87 MG, GBL-28, and GBL-37; $3 \times 10^{5}$ cells in $5 \mu \mathrm{L}$ ) were stereotactically injected into the brain parenchyma at a depth of $3 \mathrm{~mm}$. At $4-6$ weeks after the injection of the tumor cells, thin sections of the mouse brain $(10 \mu \mathrm{m})$ were processed for hematoxylin and eosin (H\&E) staining and immunofluorescence staining for GFAP.

\section{Immunofluorescence}

Thin sections of mouse brain and eyeball were washed with PBS, permeabilized with PBS containing $0.05 \%(\mathrm{v} / \mathrm{v})$ saponin and $5 \%(\mathrm{v} / \mathrm{v})$ normal goat serum for $3 \mathrm{~min}$, and treated with PBS containing 1.5\% normal goat serum for $1 \mathrm{~h}$ to block nonspecific binding. Then, the sections were labeled with an anti-GFAP antibody (1:100; cat. no. M0761 or Z0334, Dako), anti-human mitochondria antibody (1:100; cat. no. MAB1273, Millipore or cat. no. PA5-29550, Life Technologies), anti-vimentin antibody (1:100; cat. no. ab11256, Abcam), anti-nestin antibody (1:100; cat. no. MAB5326, Millipore), and anti-oligodendrocyte transcription factor 2 (OLIG2; 1:100; cat. no. sc-293163, Santa Cruz) overnight at $4{ }^{\circ} \mathrm{C}$ and treated with the corresponding Alexa Fluor 488- or 594-conjugated IgG antibody (1:500; cat. no. A11008, A11029, A11032, A11037, A11055, and A21207, Life Technologies) for $1 \mathrm{~h}$. Nuclear staining was performed using 4,6-diamidino-2-phenylindole dihydrochloride (DAPI, Sigma-Aldrich). Then, the slides were observed under a fluorescence microscope (Leica).

\section{Intravitreal injection of glioblastoma cells}

Patient-derived glioblastoma cells $\left(1 \times 10^{5}\right.$ cells $)$ were injected into the vitreous cavity of 6-week-old male Balb/c nude mice. Beginning at 2 weeks after injection, eyeballs were examined daily to monitor tumor formation. A visual grading system was employed to grade the degree of tumor formation from 0-5: grade 0 (no tumor formation), grade 1 (streak-like tumor), grade 2 (plaque-like tumor), grade 3 (definite mass formation), grade 4 (vitreous-filling tumor), and grade 5 (accompanying globe enlargement or eyeball rupture), according to the standard photographs in a previous publication ${ }^{18}$. Two independent observers (D.H. Jo and C.S. Cho) compared the observed tumor 
formation with the standard photographs. There was no disagreement in the grading in this study.

\section{Immunocytochemistry}

Glioblastoma cells were seeded in 4-well chamber slides (Nunc) and stabilized overnight. The cells were fixed with $1 \%$ paraformaldehyde at $4{ }^{\circ} \mathrm{C}$ for $10 \mathrm{~min}$ and permeabilized with $0.1 \%$ Triton X-100 solution (cat. no. T8787, Sigma-Aldrich) at room temperature for $3 \mathrm{~min}$. After treatment with $1 \%$ bovine serum albumin to minimize nonspecific binding, the cells were labeled with an anti-GFAP antibody, anti-human mitochondria antibody, anti-vimentin antibody, and anti-nestin antibody at $4{ }^{\circ} \mathrm{C}$ overnight and treated with the corresponding Alexa Fluor 488- or 594- conjugated IgG antibody (1:500; cat.no. A11008, A11029, A11032, A11037, A11055, and A21207, Life Technologies) for 1 h. Nuclear staining was performed using DAPI. Then, the slides were observed under a fluorescence microscope (Leica).

\section{Statistics}

Statistical analyses were performed using GraphPad Prism (GraphPad Software). A log-rank test was performed to detect statistically significant differences among the groups that underwent orthotopic transplantation of GBL-28, GBL-37, or U-87 MG cells. A $P$-value $<0.05$ was considered statistically significant.

\section{Results}

Isolation and preparation of tumor cells from a patient with primary and recurrent glioblastoma

In a 39-year-old female patient whose initial symptom was a short-term memory defect for 2 weeks, glioblastoma was diagnosed by stereotactic brain biopsy (Fig. 1a). Then, the patient underwent surgical tumor removal and concurrent chemoradiation therapy with temozolomide. Three months after the initial surgery, another surgical tumor removal was performed to control the regrowing tumors. Histological examination revealed that both the primary and recurrent tumors were WHO grade IV

\section{a}
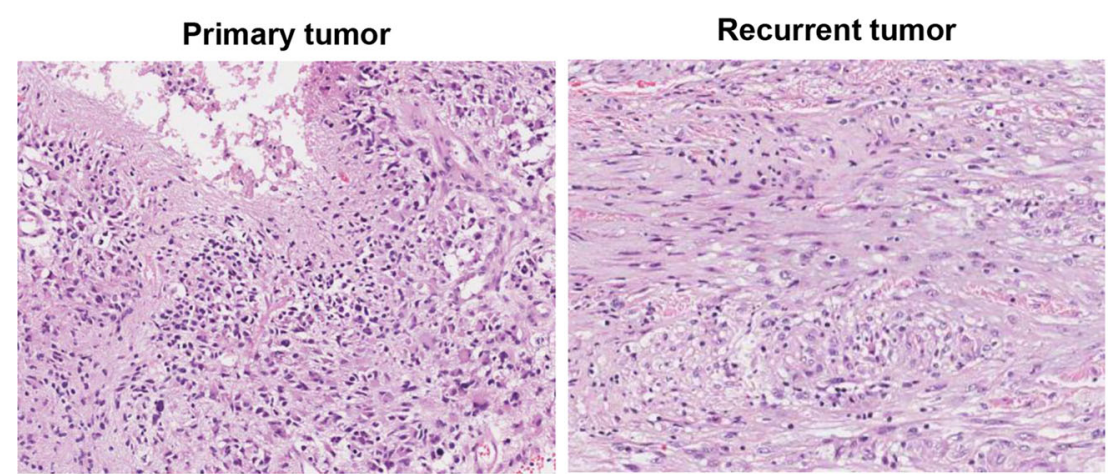

b Stereotactic biopsy

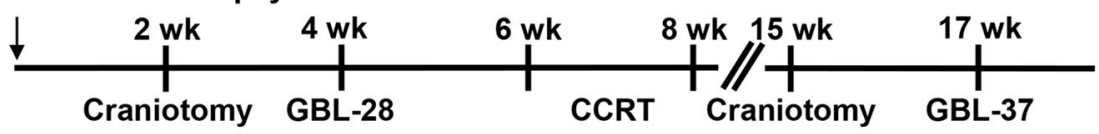

C

GBL-28

GBL-37
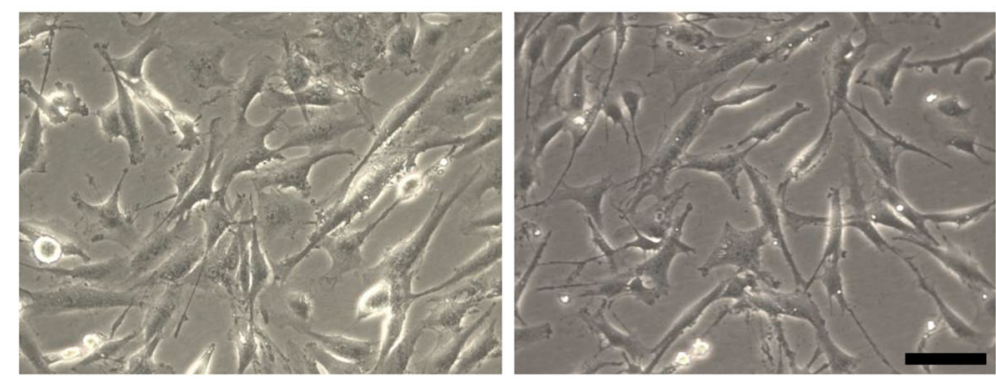

Fig. 1 Isolation and culture of tumor cells from a patient with primary and recurrent glioblastoma. a Representative images of the H\&Estained sections of the primary and recurrent tumors (original magnification, 50x). b A schematic schedule of the stereotactic biopsy, craniotomy, concurrent chemoradiation therapy (CCRT), and preparation of the patient-derived cells (GBL-28 and GBL-37 cells). wk, week(s) after initial diagnosis and stereotactic biopsy. c Representative images of GBL-28 and GBL-37 cells. Scale bar: $100 \mu \mathrm{m}$ 
Table 1 Histological and immunohistochemical characteristics of primary and recurrent glioblastoma tumors

\begin{tabular}{lll}
\hline Characteristics & Primary tumor & Recurrent tumor \\
\hline WHO grade & IV/IV & IV/IV \\
Increased cellularity & Present & Present \\
Nuclear polymorphism & Present & Present \\
Mitosis (pHH3) & 25/10 HPF & $27 / 10 \mathrm{HPF}$ \\
Vascular endothelial & Present & Absent \\
hyperplasia & & \\
Necrosis & Present & Present \\
Immunohistochemistry & & \\
GFAP & Positive & Positive \\
Vimentin & Positive & Positive \\
Nestin & Positive & Positive \\
\hline
\end{tabular}

HPF high-power field, $p H H 3$ phosphohistone $\mathrm{H} 3$

glioblastoma (Table 1). Furthermore, both tumors were positive for GFAP, vimentin, and nestin by immunohistochemical analysis (Table 1). At each surgery, tumor tissue samples were prepared for primary culture, and the glioblastoma cells were designated GBL-28 (from the primary tumor) and GBL-37 (from the recurrent tumor), respectively (Fig. 1b). Both cell lines exhibited morphological characteristics of glial cells (Fig. 1c).

\section{Unreliability of orthotopic transplantation of patient- derived glioblastoma cells}

Although orthotopic transplantation has been repeatedly performed by researchers, including our group ${ }^{2,19-22}$, some patient-derived cells formed intracranial tumors after more than 6 weeks or failed to develop tumors. When $3 \times 10^{5}$ U-87 MG, GBL-28, or GBL-37 cells were injected into the striatum of Balb/c nude mice, the survival patterns were quite different between the group injected with U-87 MG cells and the groups injected with the patient-derived glioblastoma cells $(P$-value $<0.0001$; Fig. 2a). Histological and immunohistochemical examinations demonstrated that the U-87 MG cells formed well-demarcated intracranial tumors (Fig. 2b, c, left), while the GBL-28 and GBL-37 cells did not establish any tumors, even at 6 weeks after intracranial injection (Fig. 2b, c, middle and right).

\section{Development of a novel PDX model of glioblastoma via intravitreal injection}

Intravitreal injection is a method used to establish orthotopic models of retinoblastoma ${ }^{18}$ and to deliver therapeutic agents to the retinal neuronal tissue ${ }^{23}$. Intravitreally administered cells primarily formed intraocular tumors in the vitreous cavity between the lens and retina (Fig. 3a). Then, the tumors could expand to the anterior chamber (between the cornea and lens) to occupy the entire eyeball. Because the vitreous cavity and retina of $\mathrm{Balb} / \mathrm{c}$ nude mice can be observed with the naked eye or via an indirect ophthalmoscope, the degree of tumor formation is graded with a simple visual grading system ${ }^{18}$. According to the visual grading system for intraocular xenograft tumors, grades 1-5 indicate streak-like tumors, plaque-like tumors, definite mass formation, vitreous cavity-filling tumors, and eyeball enlargement, respectively $^{18}$. Interestingly, there was a differential pattern of tumor formation between the injection of cells treated under only normal conditions (Fig. $3 \mathrm{~b}$ ) and the injection of those treated under hypoxic conditions $\left(1 \% \mathrm{O}_{2}\right)$ for $4 \mathrm{~h}$ (Fig. 3c). The cells injected after culture under normal conditions exhibited variable tumor formation (Fig. 3b). In contrast, hypoxia treatment resulted in stable and consistent mass formation (Fig. 3c). It is also remarkable that both the GBL-28 and GBL-37 cells formed tumors extending from the vitreous cavity across the retina after hypoxia treatment (Figs. 3d and 4a), and these tumors demonstrated the invasive features of glioblastoma cells. Additional experiments using four different patientderived cell lines also demonstrated that the injected cells effectively formed a tumor mass at 4 weeks after injection (Suppl. Figs. 1 and 2; Suppl. Tables 1 and 2).

\section{Immunohistochemical characterization of PDX tumors in the vitreous cavity}

The original tumors from which GBL-28 and GBL-37 cells were isolated were positive for GFAP, vimentin, and nestin expression (Table 1). Based on these data, immunohistochemical characterization was performed on PDX tumors in the vitreous cavity to identify their similarity to original tumors and to confirm that they came from the injected tumor cells, not from the mouse tissue. As expected, the tumors inside the vitreous cavity and outside the retina were positive for GFAP, vimentin, and nestin expression and human mitochondria (Fig. 4a). All the markers demonstrated cytoplasmic staining patterns, indicating their intracellular locations (Fig. 4b). There were no positive signals in the uninjected eyes (Suppl. Fig. 3). In addition, the tumors stained positive for OLIG2, one of the specific markers for the glial cell lineage (Suppl. Fig. 4$)^{24}$.

\section{Isolation and characterization of the tumor cells from PDX tumors in the vitreous cavity}

To further identify the characteristics of PDX tumors in the vitreous cavity, the tumors were isolated from the eyeballs and prepared for primary culture. As shown in Fig. 5a, the isolated cells retained the morphological characteristics of their parental cells. Further 


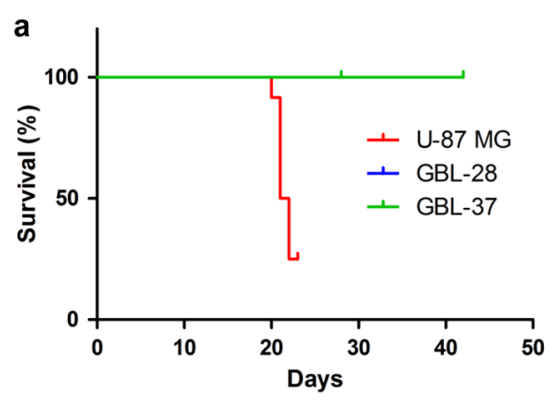

b

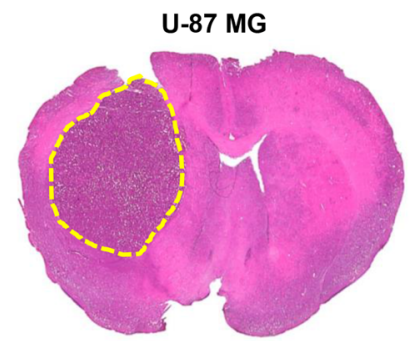

C

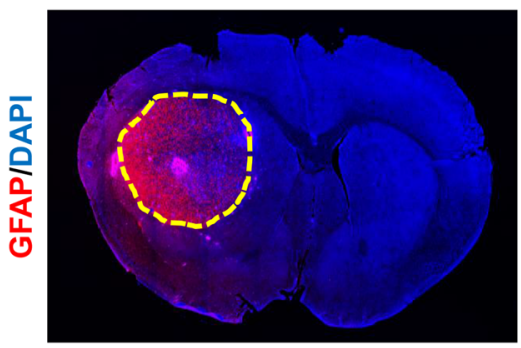

GBL-28

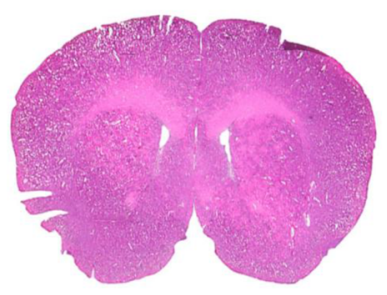

GBL-28

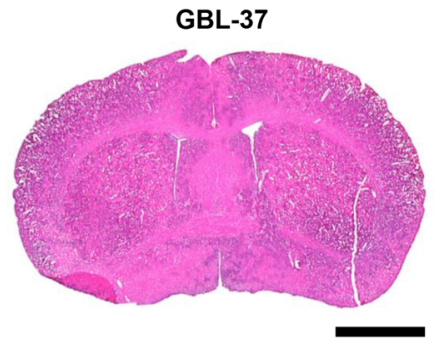

GBL-37
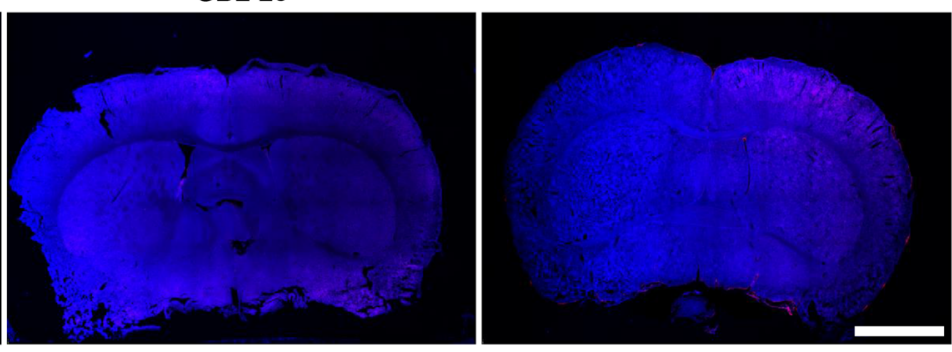

Fig. 2 Unreliability of the orthotopic transplantation of patient-derived glioblastoma cells. a Kaplan-Meier survival curves of mice $(n=12)$ intracranially injected with U-87 MG, GBL-28, or GBL-37 cells. The curve for the GBL-28 group was moved upward by $2 \%$ to prevent overlap. b Representative images of H\&E-stained sections of the brain tissue of mice at 4-6 weeks after the intracranial injection of U-87 MG (4 weeks), GBL-28 ( 6 weeks), or GBL-37 (6 weeks) cells. The yellow dashed lines indicate the intracranial tumors. Scale bar: $2 \mathrm{~mm}$. c Representative images of brain sections stained using DAPI and an antibody specific for GFAP at 4-6 weeks after the intracranial injection of U-87 MG (4 weeks), GBL-28 (6 weeks), or GBL-37 (6 weeks) cells. The yellow dashed lines indicate the intracranial tumors. Scale bar: $2 \mathrm{~mm}$

immunocytochemical analyses demonstrated that they exhibited similar positivity for GFAP (Fig. 5b), vimentin (Suppl. Fig. 5), and nestin (Suppl. Fig. 6).

\section{Discussion}

In this study, a novel PDX model of glioblastoma was established via the intravitreal injection of tumor cells. It is remarkable that the patient-derived glioblastoma cells that did not form intracranial tumors at 6 weeks after injection effectively formed intraocular tumors at 4 weeks. Further experiments using four additional patient-derived cell lines showed that they also formed intraocular tumors. The confined anatomical structure might help the development of PDX tumors in vivo in the vitreous cavity. The intraocular tumors evolved from plaque-like tumors (week 2) to a certain mass (week 4), showing efficient tumor formation and development. Further immunohistochemical characterization and tumor cell isolation experiments demonstrated that the PDX tumors retained characteristics of the original tumors.

There are already established PDX models of glioblastoma generated through subcutaneous or intracranial injection ${ }^{1-8}$. Subcutaneously administered glioblastoma tumors are confined to the subcutaneous tissue, which is quite different from the tumor microenvironment. Furthermore, subcutaneous tumors are relatively easily accessible to therapeutic agents through systemic administration, unlike brain tumors, which are protected by the blood-brain barrier. In contrast, intracranial PDX models often take $>45$ days to evaluate the efficacy of therapeutic agents ${ }^{6-8,25-27}$, and some patients-derived cells fail to form intracranial tumors, as in this study ${ }^{2}$. Additionally, even though intracranial tumors develop efficiently, magnetic resonance imaging or bioluminescence imaging are required to monitor tumor formation $^{19,28-31}$. 

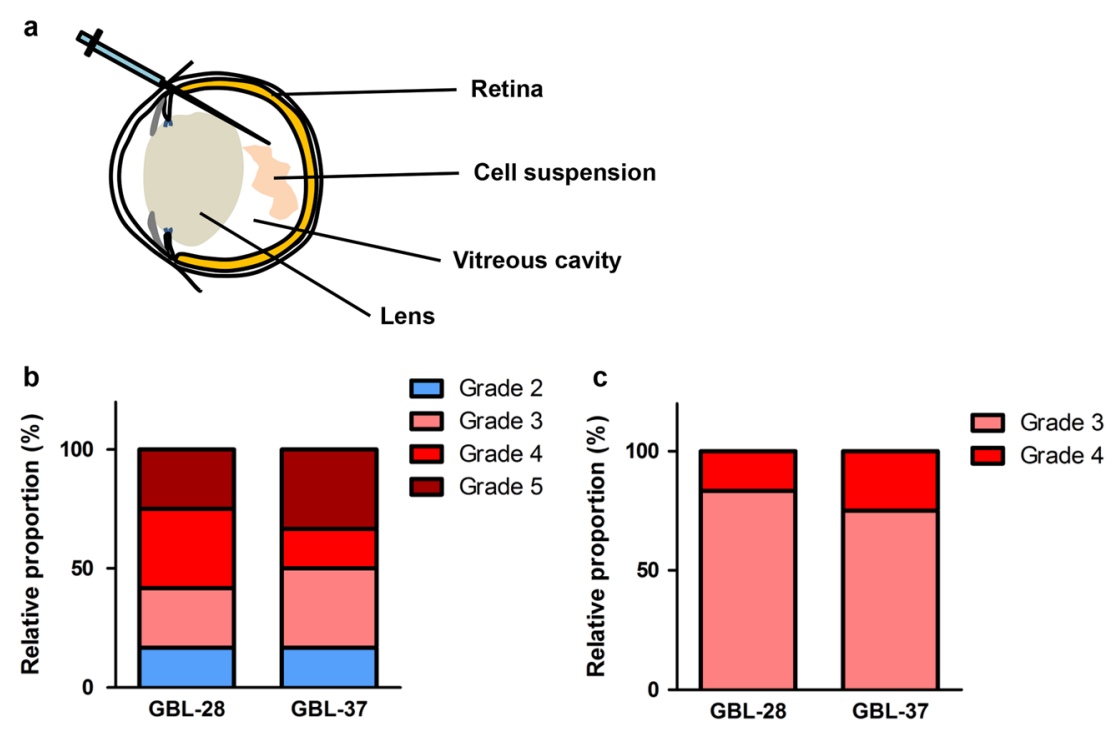

d GBL-28

GBL-37

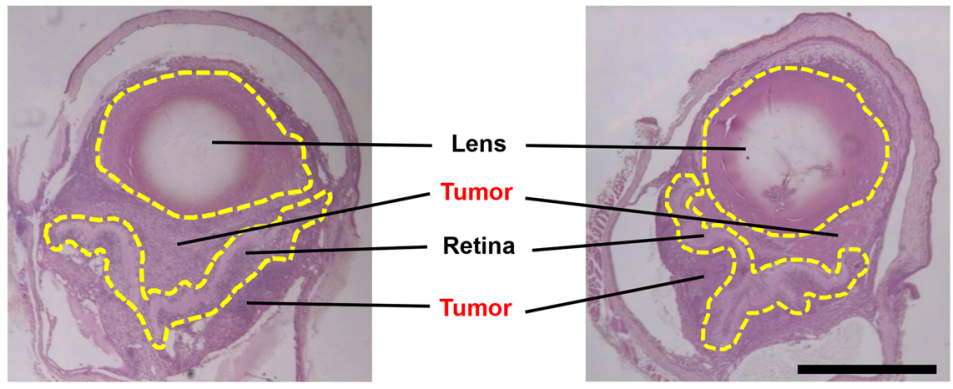

Fig. 3 Development of a novel PDX model of glioblastoma via intravitreal injection. a A schematic diagram demonstrating the intravitreal injection of tumor cells. $\mathbf{b}$ The relative proportion of mice with grade 2-5 disease after the intravitreal injection of GBL-28 and GBL-37 cells that underwent normal culture conditions. c The relative proportion of mice with grade 3 or 4 disease after the intravitreal injection of GBL-28 and GBL-37 cells that underwent hypoxia treatment for $4 \mathrm{~h}$ before injection. $\mathbf{d}$ Representative images of H\&E-stained sections of the eyeball at 4 weeks after the intravitreal injection of GBL-28 or GBL-37 cells that underwent hypoxia treatment for $4 \mathrm{~h}$ before injection. The yellow dashed lines indicate the lens and retina. Scale bar: $1 \mathrm{~mm}$

In this context, the PDX model established via intravitreal injection has several advantages. First, patientderived glioblastoma cells that underwent hypoxia treatment consistently formed an intraocular mass that retained characteristics of the original tumors regarding marker expression and invasiveness within 4 weeks. Because plaque-like tumor formation was observed at 2 weeks, therapeutic options can be screened beginning at 2 weeks after injection through systemic or direct intraocular administration. Second, the retina, which is a part of the central nervous system, mimics the microenvironment of the original tumors. Stacked neuronal cells with dynamic synapses and the blood-retinal barrier system in the retinal vasculature make the retina an effective alternative to the brain. Third, compared to intracranial tumors, intraocular tumors are easily monitored through direct observation with the naked eye or an indirect ophthalmoscope with a simple optical lens (such as a 78 diopter lens). Because no additional imaging systems are required, it is easier to perform screening and monitor tumor formation. Additionally, the visual grading system provides semiquantitative scale data for quantitative analyses of tumor formation, as in this study. It is also noteworthy that hypoxia treatment, which affects the proliferation and invasion of glioblastoma cells, increased the consistency of tumor formation ${ }^{32}$.

The median survival of patients with glioblastoma is less than 15 months $^{9-11}$. Therefore, an efficient and rapid screening system is required to screen second-line treatment options in patients with tumors resistant to conventional treatment. Although there have been several attempts to make a considerable reference library of glioblastoma with thorough characterization, including genome sequencing ${ }^{2,33}$, these efforts will be more effective with a system that can screen fresh patient-derived tumor cells more efficiently. The PDX model established via 


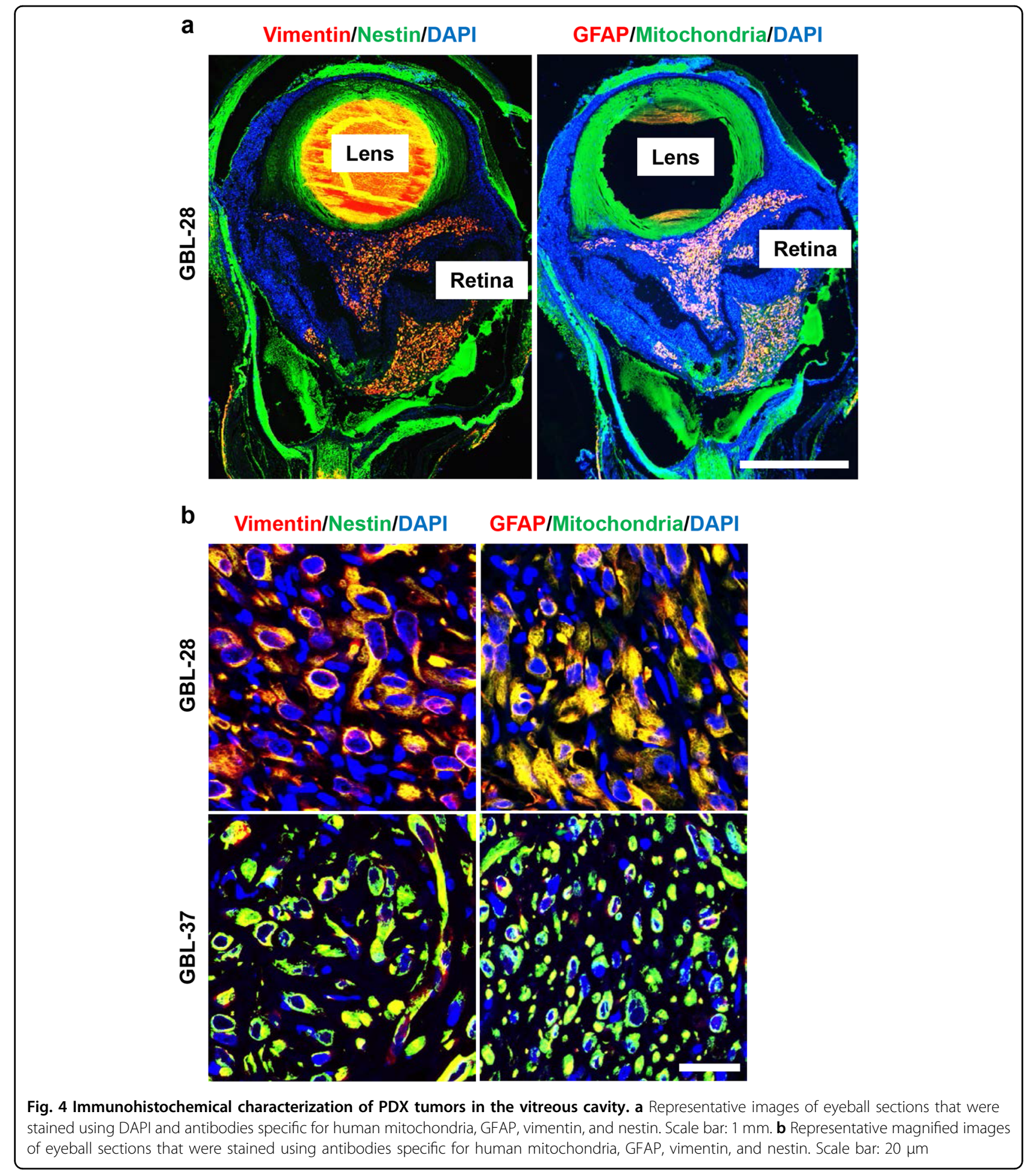

intravitreal injection is expected to screen therapeutic options within 4 weeks, which is similar to the timeframe for an orthotopically transplanted xenograft model of retinoblastoma ${ }^{18,34}$. Because relatively fewer cells $(5-10 \times$ $10^{4}$ cells) are required per mouse, several options can be evaluated simultaneously. Considering the usual preparation time for patient-derived cells ( $\sim 2$ weeks), all procedures can be completed in 6 weeks, which is much shorter than the median time from initial surgery to recurrence $^{35}$. Accordingly, this model can be complementary to the intracranial model of orthotopic transplantation. 


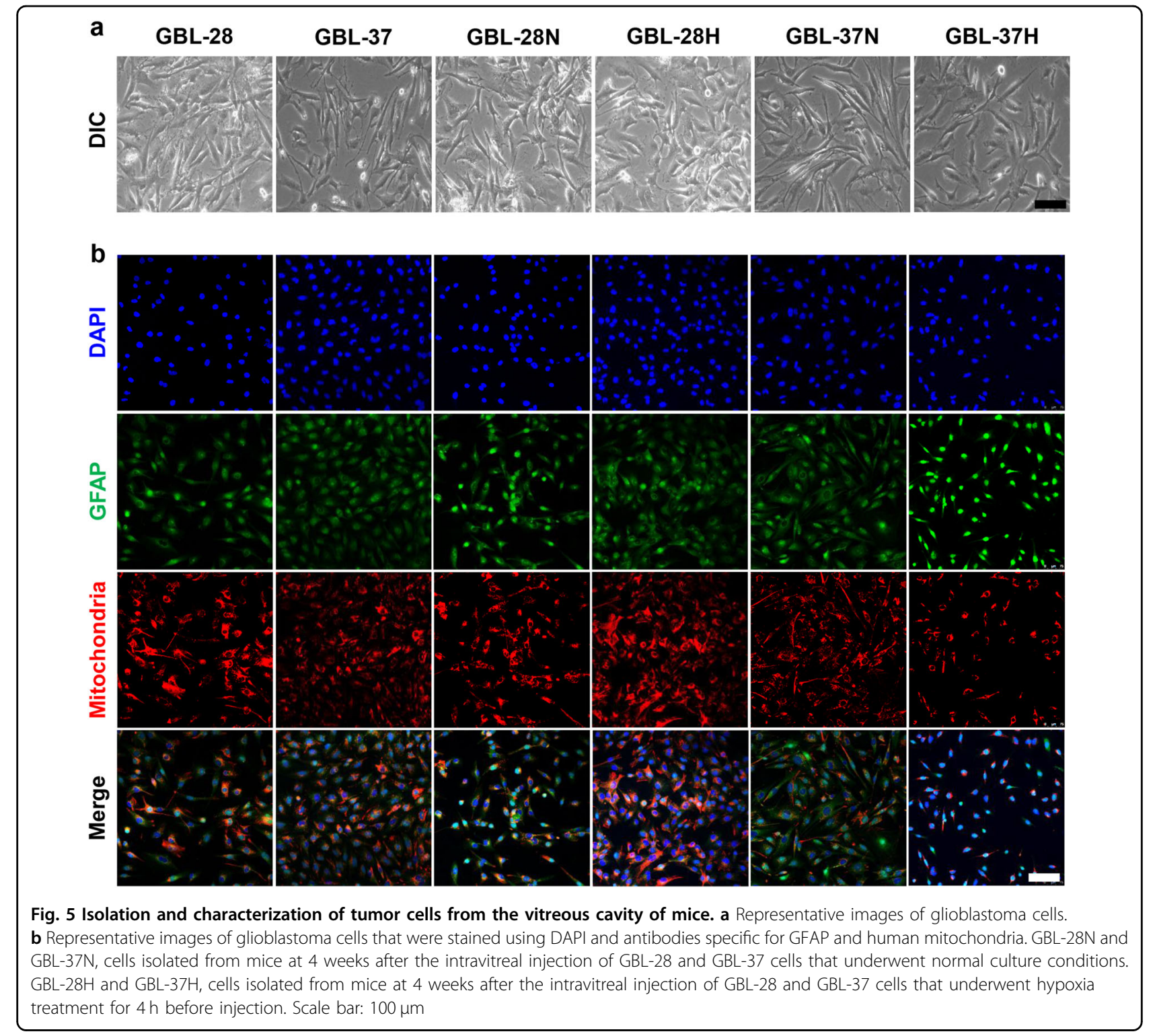

In summary, the PDX model of glioblastoma established through intravitreal injection provides an easy and efficient way to screen for tumor formation. As glioblastoma is the most common and aggressive cancer in the brain, it is imperative to develop novel therapeutic options beyond the current chemoradiation treatment. This model, which can be used 4 weeks after tumor cell injection, will be an essential tool to investigate and develop therapeutic alternatives for the treatment of glioblastoma.

\section{Acknowledgements}

This study was supported by the Korea Healthcare Technology R\&D Project (grant no. HI11C21100200) funded by the Ministry of Health \& Welfare,

Republic of Korea; the Technology Innovation Program (grant no. 10050154, Business Model Development for Personalized Medicine Based on Integrated Genome and Clinical Information) funded by the Ministry of Trade, Industry \& Energy (MI, Korea); the Bio \& Medical Technology Development Program of the NRF funded by the Korean government, MSIP (grant no. 2015M3C7A1028926); the National Research Foundation of Korea Grant funded by the Ministry of Science and ICT (grant no. NRF-2017M3C7A1047392); the Bio \& Medical Technology Development Program of the National Research Foundation and MSIP (NRF-2015M3A9E6028949); the Global Core Research Center (GCRC) grant from NRF/MEST, Republic of Korea (2012-0001187); the Creative Materials Discovery Program through the National Research Foundation of Korea (NRF) funded by the Ministry of Science and ICT (2018M3D1A1058826); and the Basic Science Research Program through the National Research Foundation of Korea (NRF) funded by the Ministry of Education (2017R1A6A3A04004741).

\section{Author details}

'Department of Neurosurgery, Seoul National University Hospital, Seoul 03080, Republic of Korea. ${ }^{2}$ Fight against Angiogenesis-Related Blindness (FARB) Laboratory, Clinical Research Institute, Seoul National University Hospital, Seoul 03080, Republic of Korea. ${ }^{3}$ Tumor Microenvironment Research Center, Global Core Research Center, Seoul National University, Seoul 08826, Republic of Korea. ${ }^{4}$ Department of Ophthalmology, Seoul National University College of Medicine, Seoul 03080, Republic of Korea. ${ }^{5}$ Department of Neurosurgery, Seoul National University College of Medicine, Seoul 03080, Republic of Korea. ${ }^{6}$ Department of Biomedical Sciences, Seoul National University College of 
Medicine, Seoul 03080, Republic of Korea. ${ }^{7}$ Cancer Research Institute, Seoul National University College of Medicine, Seoul 03080, Republic of Korea. ${ }^{8}$ Hypoxia Ischemia Disease Institute, Seoul National University College of Medicine, Seoul 03080, Republic of Korea

\section{Author contributions}

J.L. drafted the manuscript and performed in vitro experiments. D.H.J. drafted the manuscript and performed the intravitreal injections and further analyses. J.H.K. performed analyses of the in vivo data. C.S.C. performed the intravitreal injections and further processing of the ocular tissue samples. J.E.H., Y.K., and H.P. performed orthotopic transplantation experiments. S.H.Y. and Y.S.Y. supervised the intravitreal injections and further analyses. H.E.M., H.R.P. and D.G.K. supervised the orthotopic transplantations and further analyses. J.H.K. and S.H.P. designed the study and drafted the manuscript.

\section{Conflict of interest}

The authors declare that they have no conflict of interest.

\section{Publisher's note}

Springer Nature remains neutral with regard to jurisdictional claims in published maps and institutional affiliations.

Supplementary information accompanies this paper at https://doi.org/ 10.1038/s12276-019-0241-3.

Received: 11 July 2018 Revised: 31 December 2018 Accepted: 2 January 2019.

Published online: 16 April 2019

\section{References}

1. Verreault, M. et al. Preclinical Efficacy of the MDM2 Inhibitor RG7112 in MDM2Amplified and TP53 Wild-type Glioblastomas. Clin. Cancer Res. 22, 1185-1196 (2016).

2. Joo, K. M. et al. Patient-specific orthotopic glioblastoma xenograft models recapitulate the histopathology and biology of human glioblastomas in situ. Cell Rep. 3, 260-273 (2013).

3. Xia, S. et al. Tumor microenvironment tenascin- $\mathrm{C}$ promotes glioblastoma invasion and negatively regulates tumor proliferation. Neuro. Oncol. 18, 507-517 (2016)

4. Yu, D. et al. Multiplexed RNAi therapy against brain tumor-initiating cells via lipopolymeric nanoparticle infusion delays glioblastoma progression. Proc. Natl Acad. Sci. USA 114, E6147-E6156 (2017).

5. Tivnan, A. et al. Anti-GD2-ch14.18/CHO coated nanoparticles mediate glioblastoma (GBM)-specific delivery of the aromatase inhibitor, Letrozole, reducing proliferation, migration and chemoresistance in patient-derived GBM tumor cells. Oncotarget 8, 16605-16620 (2017).

6. Ramachandran, M. et al. Safe and effective treatment of experimental neuroblastoma and glioblastoma using systemically delivered triple microRNAdetargeted oncolytic semliki forest virus. Clin. Cancer Res. 23, 1519-1530 (2017).

7. MCNeill, R. S. et al. Combination therapy with potent PI3K and MAPK inhibitors overcomes adaptive kinome resistance to single agents in preclinical models of glioblastoma. Neuro. Oncol. 19, 1469-1480 (2017).

8. Canella, A. et al. Efficacy of onalespib a long-acting second generation HSP9O inhibitor as a single agent and in combination with temozolomide against malignant gliomas. Clin. Cancer Res. 23, 6215-6226 (2017).

9. Johnson, D. R. \& O'Neill, B. P. Glioblastoma survival in the United States before and during the temozolomide era. J. Neurooncol. 107, 359-364 (2012).

10. Stupp, R. et al. Effects of radiotherapy with concomitant and adjuvant temozolomide versus radiotherapy alone on survival in glioblastoma in a randomised phase III study: 5-year analysis of the EORTC-NCIC trial. Lancet Oncol. 10, 459-466 (2009).
11. Stupp, R. et al. Radiotherapy plus concomitant and adjuvant temozolomide for glioblastoma. N. Engl. J. Med. 352, 987-996 (2005).

12. Jo, D. H., Kim, J. H. \& Kim, J. H. How to overcome retinal neuropathy: the fight against angiogenesis-related blindness. Arch. Pharm. Res. 33, 1557-1565 (2010).

13. Kim, J. H. et al. Blood-neural barrier: intercellular communication at gliovascular interface. J. Biochem. Mol. Biol. 39, 339-345 (2006).

14. Abbott, N. J., Ronnback, L. \& Hansson, E. Astrocyte-endothelial interactions at the blood-brain barrier. Nat. Rev. Neurosci. 7, 41-53 (2006).

15. Matias, D. et al. Microglia-glioblastoma interactions: new role for Wnt signaling. Biochim. Biophys. Acta 1868, 333-340 (2017).

16. Poon, C. C., Sarkar, S., Yong, V. W. \& Kelly, J. J. P. Glioblastoma-associated microglia and macrophages: targets for therapies to improve prognosis. Brain 140, 1548-1560 (2017).

17. Silver, D. J., Sinyuk, M., Vogelbaum, M. A., Ahluwalia, M. S. \& Lathia, J. D. The intersection of cancer, cancer stem cells, and the immune system: therapeutic opportunities. Neuro Oncol. 18, 153-159 (2016).

18. Jo, D. H. et al. L1 increases adhesion-mediated proliferation and chemoresistance of retinoblastoma. Oncotarget 8, 15441-15452 (2017).

19. Cho, K. T. et al. Concurrent treatment with BCNU and Gamma Knife radiosurgery in the rat malignant glioma model. J. Neurol. Surg. A Cent. Eur. Neurosurg. 73, 132-141 (2012)

20. Han, T. J. et al. Inhibition of STAT3 enhances the radiosensitizing effect of temozolomide in glioblastoma cells in vitro and in vivo. J. Neurooncol. $\mathbf{1 3 0}$ 89-98 (2016).

21. Kang, S. et al. Trifluoperazine, a well-known antipsychotic, inhibits glioblastoma invasion by binding to calmodulin and disinhibiting calcium release channel IP3R. Mol. Cancer Ther. 16, 217-227 (2017).

22. Kang, S. S. et al. Caffeine-mediated inhibition of calcium release channe inositol 1,4,5-trisphosphate receptor subtype 3 blocks glioblastoma invasion and extends survival. Cancer Res. 70, 1173-1183 (2010).

23. Jo, D. H., Kim, J. H., Kim, K. W., Suh, Y. G. \& Kim, J. H. Allosteric regulation of pathologic angiogenesis: potential application for angiogenesis-related blindness. Arch. Pharm. Res. 37, 285-298 (2014).

24. Trepant, A. L. et al. Identification of OLIG2 as the most specific glioblastoma stem cell marker starting from comparative analysis of data from similar DNA chip microarray platforms. Tumour Biol. 36, 1943-1953 (2015).

25. Arif, T. et al. VDAC1 is a molecular target in glioblastoma, with its depletion leading to reprogrammed metabolism and reversed oncogenic properties. Neuro. Oncol. 19, 951-964 (2017).

26. Mohammad, F. et al. EZH2 is a potential therapeutic target for H3K27Mmutant pediatric gliomas. Nat. Med. 23, 483-492 (2017).

27. Jiang, X. et al. Nanoparticle engineered TRAlL-overexpressing adipose-derived stem cells target and eradicate glioblastoma via intracranial delivery. Proc. Nat Acad. Sci. USA 113, 13857-13862 (2016).

28. Miller, T. E. et al. Transcription elongation factors represent in vivo cancer dependencies in glioblastoma. Nature 547, 355-359 (2017).

29. Eom, K. Y. et al. The effect of chemoradiotherapy with SRC tyrosine kinase inhibitor, PP2 and temozolomide on malignant glioma cells in vitro and in vivo. Cancer Res. Treat. 48, 687-697 (2016).

30. Ugolkov, A. et al. Combination treatment with the GSK-3 inhibitor 9-ING-41 and CCNU cures orthotopic chemoresistant glioblastoma in patient-derived xenograft models. Transl. Oncol. 10, 669-678 (2017).

31. Lee, T. J. et al. RNA nanoparticle as a vector for targeted siRNA delivery into glioblastoma mouse model. Oncotarget 6, 14766-14776 (2015).

32. Monteiro, A. R., Hill, R., Pilkington, G. J. \& Madureira, P. A. The role of hypoxia in glioblastoma invasion. Cells 6, 45 (2017).

33. Oh, Y. T. et al. Translational validation of personalized treatment strategy based on genetic characteristics of glioblastoma. PLOS ONE $\mathbf{9}$, e103327 (2014).

34. Jo, D. H. et al. STAT3 inhibition suppresses proliferation of retinoblastoma through down-regulation of positive feedback loop of STAT3/miR-17-92 clusters. Oncotarget 5, 11513-11525 (2014).

35. Kim, H. R. et al. Outcome of salvage treatment for recurrent glioblastoma J. Clin. Neurosci. 22, 468-473 (2015). 